\title{
Operative safety and oncologic outcomes in rectal cancer based on the level of inferior mesenteric artery ligation: a stratified analysis of a large Korean cohort
}

\author{
Mohammed A. AISuhaimi, Seung Yoon Yang, Jae Hyun Kang, Jamal F. AlSabilah, Hyuk Hur, Nam Kyu Kim \\ Department of Surgery, Yonsei University College of Medicine, Seoul, Korea
}

\begin{abstract}
Purpose: To compare high and low inferior mesenteric artery (IMA) ligation in a large number of patients, and investigate the short-term and long-term outcomes.

Methods: This retrospective study compared outcomes between high IMA ligation and low IMA ligation with dissection of lymph nodes (LNs) around the IMA origin. A total of 1,213 patients underwent elective low anterior resection with doublestapling anastomosis for stage I-III rectal cancer located $\geq 6 \mathrm{~cm}$ from the anal verge $(835$ patients underwent IMA ligation at the IMA origin; 378 patients underwent IMA ligation directly distal to the root of the left colic artery along with dissection of $L N s$ around the IMA origin).

Results: There was no difference in anastomotic leakage rate between groups. The 2 groups did not significantly differ in intraoperative blood loss, perioperative complications, total number of harvested LNs, and metastatic IMA LNs. However, more metastatic LNs were harvested in the high-tie than in the low-tie group $(1.3 \pm 2.9 \mathrm{vs}$. $0.8 \pm 1.9, P=0.002)$, and the incidence of positive pathologic nodal status was higher in the high-tie group (37.9\% vs. $28.6 \%, P=0.001)$. The 5 -year local recurrence-free and metastasis-free survival rates were similar between groups, as were the 5 -year overall and cancerspecific survival rates.

Conclusion: Low IMA ligation with dissection of LNs around the IMA origin showed no differences in anastomotic leakage rate compared with high IMA ligation, without affecting oncologic outcomes. High IMA ligation did not seem to increase the number of total harvested LNs, whereas the ratio of metastatic apical LNs were similar between groups.

[Ann Surg Treat Res 2019;97(5):254-260]
\end{abstract}

Key Words: High ligation, Inferior mesenteric artery, Low ligation, Rectal neoplasms, Treatment outcome

\section{INTRODUCTION}

Lymph node (LN) metastasis is a major prognostic factor in patients undergoing surgery for rectal cancer [1]. Precise LN dissection is considered to be crucial in rectal cancer surgery, and the pathologic finding of LN involvement is a significant prognostic factor for survival after low anterior resection (LAR) [2]. There is an ongoing debate among surgeons and institutions about which level of inferior mesenteric artery (IMA) ligation in rectal cancer surgery provides the best outcomes for patients [3]. The importance of the level of arterial ligation remains controversial with respect to several aspects, including the oncologic outcome, ability to perform accurate tumor staging, and perfusion of the distal colonic artery, which is correlated with anastomotic leakage (AL) [3-5].

It has been suggested that the incidence of AL after LAR
Received July 10, 2019, Revised September 24, 2019,

Accepted October 8, 2019

\section{Corresponding Author: Nam Kyu Kim}

Division of Colon and Rectal Surgery, Department of Surgery, Severance Hospital, Yonsei University College of Medicine, 50-1 Yonsei-ro, Seodaemun-gu, Seoul 03722, Korea

Tel: +82-2-2228-2100, Fax: +82-2-313-8289

E-mail:namkyuk@yuhs.ac

ORCID: https://orcid.org/0000-0003-0639-5632
- The abstract was accepted as an e-poster in the American Society of Colon and Rectal Surgeons meeting held from June 10, 2017, to June 14 , 2017, in Seattle, WA, USA.

Copyright (c) 2019, the Korean Surgical Society

(c) Annals of Surgical Treatment and Research is an Open Access Journal. All articles are distributed under the terms of the Creative Commons Attribution NonCommercial License (http://creativecommons.org/licenses/by-nc/4.0/) which permits unrestricted non-commercial use, distribution, and reproduction in any medium, provided the original work is properly cited. 
for rectal cancer depends on the level of ligation $[6,7]$. When creating an anastomosis between the proximal colon and the remaining rectum, the mesocolon must be extended to minimize the tension on the anastomosis, and division of the IMA at its origin has been reported to be useful in this respect [8]. After a high tie is performed, the perfusion to the proximal colon is supplied solely by the superior mesenteric artery; thus, decreased anastomotic perfusion is a matter of concern.

However, to date, there are few well-designed randomized controlled trials (RCTs) with reliable results. Previous retrospective studies and a recent RCT have not proved the superiority of a single approach for arterial ligation in terms of oncologic outcomes [3,9]. Therefore, the authors recommended exercising caution when choosing the level of arterial ligation $[7,10]$. We aimed to shed light on this controversial issue by investigating the short-term and long-term outcomes of both procedures in a large sample of patients who underwent LAR with double-stapling anastomosis.

\section{METHODS}

\section{Patients and data collection}

We performed a retrospective study using the prospective electronic records of 1,213 patients with rectal cancer treated with elective LAR with double-stapling colorectal anastomosis at Severance Hosptial, Yonsei University Health System, from January 2007 to March 2013. The study protocol was approved by the Institutional Review Board (IRB) of Yonsei University (IRB No 2019-1183-001). Written informed consents were waived because of being a retrospective study. We included patients with stage I-III, histologically proven, invasive adenocarcinoma of the rectum, located $6-15 \mathrm{~cm}$ from the anal verge. We excluded patients with stage IV rectal cancer; those with multiple, histologically distinct primary tumors; and those who underwent procedures other than double-stapling anastomosis, including abdominoperineal resection, LAR with coloanal anastomosis, or intersphincteric resection. We divided the participants into 2 groups for analysis. The first group included 835 patients who underwent high IMA ligation at the root of the IMA (high tie). The second group included 378 patients who underwent low IMA ligation directly distal to the origin of the left colic artery (LCA) along with dissection of the LNs around the root of the IMA (low tie). The selection of the level of IMA ligation was decided by the surgeons.

\section{Evaluation parameters}

The primary outcome of this study was the AL rate. The secondary outcomes were long-term oncologic outcomes, including local recurrence-free survival rate, metastasis-free survival rate, overall survival, and cancer-specific survival, of the high-tie and low-tie groups. We defined AL as the breakdown of the colorectal anastomosis with an infected fluid collection in the pelvic cavity within 60 days after the index operation. AL was diagnosed using CT or on the basis of clinical symptoms and signs including change of drain color and/or fever with peritonitis. Overall survival was defined as the duration from the date of surgery to the date of death. Cancer specific survival was defined as the time between surgery and cancerrelated death. Local recurrence was defined as recurrence at the primary tumor site confirmed on radiologic or histologic examination. Recurrence beyond the primary site was considered distant metastasis.

The variables that were analyzed and compared between patients with high and low IMA ligation were as follows: age, sex, American Society of Anesthesiologists physical status classification grade, preoperative CEA level, surgical approach (open surgery or minimal invasive surgery), neoadjuvant chemoradiotherapy, and operative outcomes (e.g., operative time and intraoperative blood loss). Pathologic staging was based on the 7th edition of the American Joint Commission on Cancer TNM system [11]. Surgical specimens were analyzed to determine the number of harvested LNs and the circumferential resection margin (CRM). CRM involvement was defined as the presence of tumor cells within $1 \mathrm{~mm}$ of the CRM [12]. The tumor size, proximal resection margin, and distal resection margin were determined in the operating room, and pathologic reports were reviewed to obtain additional information.

\section{Perioperative management}

The preoperative staging workup included colonoscopy with biopsy; CEA testing; chest, abdominal, and pelvic CT; and rectal MRI. Neoadjuvant chemoradiotherapy was administered in patients with T3/4 midrectal cancer or positive LNs. We used a standard, long-course, neoadjuvant regimen of 5-fluorouracilbased chemotherapy and a total dose of 50.4 Gy of externalbeam radiation. The follow-up strategies for patients were identical between the groups. The patients were followed up every 6 months for the first 3 years after surgery and yearly thereafter. Each follow-up evaluation included medical history taking, physical examination, and measurement of serum CEA level. Routine imaging studies consisted of chest radiography and $\mathrm{CT}$ of the chest, abdomen, and pelvis. Chest radiography and abdominopelvic CT were performed 6 months after surgery for 3 years and annually thereafter. Colonoscopy was performed annually after surgery. Ultrasonography, whole-body bone scintigraphy, and PET were performed when there was suspicion of recurrence on routine imaging studies.

\section{Surgical technique}

All surgeries were performed by five surgeons of the Division of Colorectal Surgery of Severance Hosptial, Yonsei University Health System. All procedures started from a medial to a 
lateral approach according to the oncologic concept. The level of ligation of the IMA was at the origin of the aorta among patients allocated to high ligation, and below the origin of the LCA among those allocated to low ligation (Fig. 1). Before 2010, high IMA ligation was the standard practice used by all surgeons involved in this study. Starting in 2010, three of the surgeons began performing low IMA ligation with concurrent dissection of the LNs around the root of the IMA for rectal cancer surgeries, whereas the other 2 surgeons continued to perform high ligation for all rectal cancer surgeries. However, these 3 surgeons selectively performed high ligation when preoperative APCT showed suspicious metastatic LNs at the root of the IMA or when metastasis LNs were diagnosed by frozen biopsy at the root of IMA. The peritoneum was incised at the level of the sacral promontory below the aortic bifurcation. Medial-to-lateral dissection of the sigmoid and descending colon along the avascular plane with autonomic nerve preservation and dissection of the LNs around the root of the IMA were performed. For low ligation, after identifying the LCA around the origin of the IMA, the IMA was resected below the origin of the LCA. Then, the inferior mesenteric vein was ligated with careful skeletonization on the level of its origin after assessing the collateral artery near the inferior mesenteric vein and pancreas. The left paracolic gutter was dissected, and the greater omentum of the transverse colon was detached. Complete splenic flexure mobilization was performed for a tension-free anastomosis for mid-rectal cancer, regardless if low or high IMA ligation was done, and the descending colon was used for the anastomosis. Patients with midrectal cancer underwent total mesorectal excision, whereas those with upper rectal cancer underwent tumor-specific mesorectal excision. After specimen extraction, end-to-end intracorporeal anastomosis was done with a double stapling technique. A suction drain was placed in the pelvic cavity. The anastomosis was then tested using an air-leak test. Protective ileostomy was performed in patients with two or more of the following indications: male sex, low anastomosis, neoadjuvant chemoradiotherapy, use of multiple staplers, and positive air-leak test. The operative details were as described in our previous studies [13,14].

Table 1. Patients' baseline and clinical characteristics

\begin{tabular}{lccc}
\hline \multicolumn{1}{c}{ Characteristic } & $\begin{array}{c}\text { High tie } \\
(\mathrm{n}=835)\end{array}$ & $\begin{array}{c}\text { Low tie } \\
(\mathrm{n}=378)\end{array}$ & P-value \\
\hline $\begin{array}{l}\text { Age }(\mathrm{yr}) \\
\text { Sex }\end{array}$ & $60.6 \pm 10.8$ & $60.2 \pm 11.5$ & 0.55 \\
$\quad$ Male & $503(66.2)$ & $241(63.8)$ & \\
$\quad$ Female & $332(33.8)$ & $137(36.2)$ & \\
Body mass index $\left(\mathrm{kg} / \mathrm{m}^{2}\right)$ & $23.4 \pm 3.2$ & $23.2 \pm 3.4$ & 0.23 \\
ASA PS classification & & & 0.246 \\
$\quad$ I-II & $608(72.8)$ & $263(69.6)$ & \\
$\quad$ IIII & $227(27.2)$ & $115(30.4)$ & \\
Preoperative CEA & $5.4 \pm 10.4$ & $5.5 \pm 13.0$ & 0.927 \\
Neoadjuvant CRT & $146(17.5)$ & $73(19.3)$ & 0.47 \\
Tumor location from AV & & & 0.122 \\
(cm) & & & \\
$\quad$ 6-10 & $453(54.3)$ & $187(49.5)$ & \\
$\quad$ 10.1-15 & $382(45.7)$ & $191(50.5)$ & \\
Operative approach & & & $<0.001$ \\
$\quad$ Open & $133(15.9)$ & $21(5.6)$ & \\
$\quad$ Laparoscopic & $443(53.1)$ & $241(63.8)$ & \\
$\quad$ Robotic & $259(31.0)$ & $116(30.7)$ & \\
Diverting ileostomy & $152(18.2)$ & $87(23.0)$ & 0.05 \\
\hline
\end{tabular}

Values are presented as mean \pm standard deviation or number (\%).

ASA PS, American Society of Anesthesiologists physical status; $\mathrm{CRT}$, chemoradiotherapy; AV, anal verge.

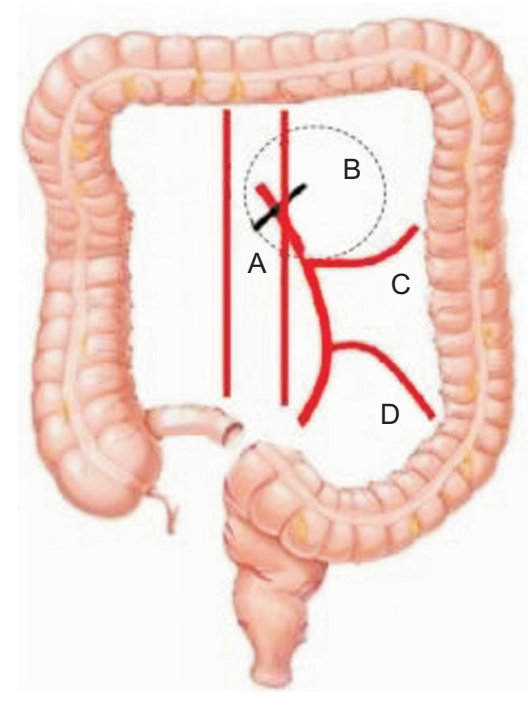

High tie

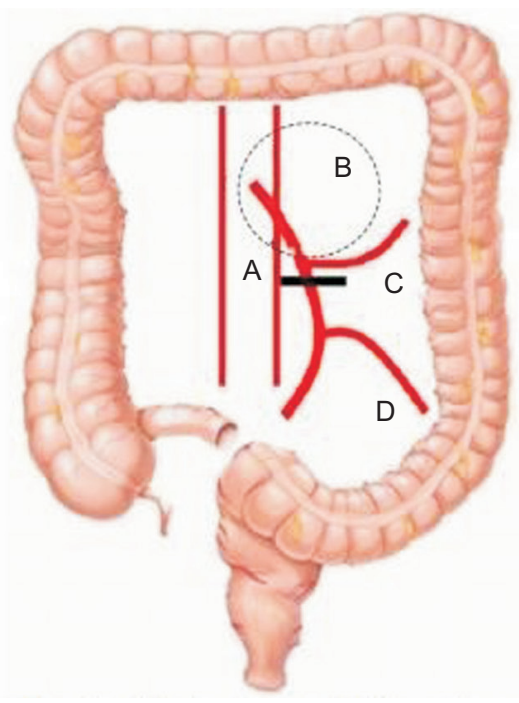

Low tie with lymph node dissection around the root of the IMA (low tie with LND)
Fig. 1. Level of ligation of the inferior mesenteric artery (IMA). (Left) High tie. (Right) Low tie with lymph node dissection around the root of the IMA (low tie with lymph node dissection [LND]). A, IMA; B, lymph node around the root of the IMA; C, left colic artery; D, sigmoid artery. 


\section{Statistical analysis}

All statistical analyses were performed using IBM SPSS Statistics ver. 23.0 (IBM Co., Armonk, NY, USA). Categorical variables were analyzed using the $\chi^{2}$ test, and continuous variables were analyzed using Student t-test. Differences in survival between groups were compared using the KaplanMeier method and tested with the log-rank test. A P-value of $<0.05$ was considered statistically significant.

\section{RESULTS}

\section{Patients' baseline and clinical characteristics}

There were no significant differences between patients who underwent high IMA ligation and those who underwent low IMA ligation with respect to age, sex, body mass index, American Society of Anesthesiologists physical status classification grade, preoperative CEA level, neoadjuvant chemoradiotherapy, tumor location from the anal verge, and diverting ileostomy. Patients who underwent high IMA ligation were more likely to have undergone an open operation than those who underwent low IMA ligation ( $15.9 \%$ vs. $5.6 \%, \mathrm{P}<0.001)$ (Table 1).

Table 2. Pathologic and surgical outcomes

\begin{tabular}{|c|c|c|c|}
\hline Variable & $\begin{array}{l}\text { High tie } \\
(\mathrm{n}=835)\end{array}$ & $\begin{array}{l}\text { Low tie } \\
(\mathrm{n}=378)\end{array}$ & P-value \\
\hline Pathologic T & & & 0.27 \\
\hline $\mathrm{pCR}$ & $62(7.4)$ & $24(6.3)$ & \\
\hline pT1 or ypT1 & $80(9.6)$ & $47(12.4)$ & \\
\hline рT2 or ypT2 & $220(26.3)$ & $97(25.7)$ & \\
\hline рT3 or ypT3 & $436(52.2)$ & $188(49.7)$ & \\
\hline pT4 or ypT4 & $24(2.9)$ & $19(5)$ & \\
\hline Pathologic N & & & 0.001 \\
\hline pNO or ypNO & 517 (61.9) & $270(71.4)$ & \\
\hline pN1 or ypN1 & $219(26.2)$ & $87(23)$ & \\
\hline $\mathrm{pN} 2$ or ypN2 & $98(11.7)$ & $21(5.6)$ & \\
\hline $\begin{array}{l}\text { Total lymph nodes } \\
\text { harvested }\end{array}$ & $17.6 \pm 9.8$ & $18.3 \pm 8.7$ & 0.37 \\
\hline $\begin{array}{l}\text { Metastatic lymph } \\
\text { nodes }\end{array}$ & $1.3 \pm 2.9$ & $0.8 \pm 1.9$ & 0.002 \\
\hline $\begin{array}{l}\text { Metastatic IMA } \\
\text { lymph nodes }\end{array}$ & $20(2.4)$ & $5(1.6)$ & 0.16 \\
\hline CRM & $56(6.7)$ & $15(4)$ & 0.07 \\
\hline Operative time (min) & $248.1 \pm 110$ & $281.8 \pm 181.4$ & $<0.001$ \\
\hline $\begin{array}{l}\text { Estimated blood } \\
\text { loss }(\mathrm{mL})\end{array}$ & $136.4 \pm 205.6$ & $155.1 \pm 181.4$ & 0.15 \\
\hline $\begin{array}{l}\text { Postoperative } \\
\text { complication rate }\end{array}$ & $40(4.8)$ & $12(3.2)$ & 0.22 \\
\hline Anastomotic leak rate & $94(11.3)$ & $41(10.8)$ & 0.85 \\
\hline
\end{tabular}

Values are presented as number $(\%)$ or mean \pm standard deviation.

pCR, pathologic complete response; IMA, inferior mesenteric artery; CRM, circumferential resection margin.

\section{Pathologic and surgical outcomes}

The pathologic and surgical outcomes of patients who underwent LAR for rectal cancer are summarized in Table 2. The total numbers of harvested LNs and metastatic IMA LNs were not significantly different between the groups. However, more metastatic LNs were harvested in the high-tie group than in the low-tie group $(1.3 \pm 2.9$ vs. $0.8 \pm 1.9, \mathrm{P}=0.002)$, and the incidence of positive pathologic $\mathrm{N}$ status was higher in the high-tie group ( $37.9 \%$ vs. $28.6 \%, \mathrm{P}=0.001$ ). The other pathologic outcomes, such as pathologic T stage and CRM involvement, showed no difference between the 2 groups. Factors related to surgical outcomes were compared between the groups. The 2 groups had similar incidences of AL (the primary end point of this study), which occurred in 94 patients (11.3\%) who underwent high IMA ligation and in 41 patients $(10.8 \%)$ who underwent low IMA ligation $(\mathrm{P}=0.85)$. Postoperative complications occurred in 40 patients $(4.8 \%)$ who underwent high IMA ligation and in 12 patients (3.2\%) who underwent low IMA ligation $(P=0.22)$. In both groups, the most common complication was paralytic ileus ( $38 \%$ and $42 \%$ ), followed by wound infection (25\% and $30 \%$ ), bleeding (27\% and $25 \%$ ), and urinary infection (10\% and $3 \%$ ). As expected, the operative time was significantly longer in the low-tie group $(248.1 \pm 110$ minutes vs. $281.8 \pm 181.4$ minutes, $P<0.001$ ), whereas there was no significant difference in estimated blood loss.

\section{Oncologic outcomes}

The oncologic outcomes of the 2 groups are summarized in Table 3. With a median follow-up of 44 months (interquartile range, 27-59 months), the local recurrence-free survival rate was comparable between groups $(92.1 \%$ vs. $96.1 \%, \mathrm{P}=0.197$ ) (Fig. 2A). In addition, there was no significant difference in the metastatic recurrence-free survival rate between high-tie and low-tie groups (Fig. 2B). As shown in Fig. $2 \mathrm{C}$ and D, there were no significant differences between groups in the 5 -year overall survival and 5-year cancer-specific survival rates.

\section{DISCUSSION}

There is considerable controversy about the level at which arterial ligation should be performed, and on the relationships between the ligation level and postoperative morbidity,

Table 3. Oncologic outcomes

\begin{tabular}{lccc}
\hline \multicolumn{1}{c}{ Variable } & $\begin{array}{c}\text { High tie } \\
(\mathrm{n}=835)\end{array}$ & $\begin{array}{c}\text { Low tie } \\
(\mathrm{n}=378)\end{array}$ & $\begin{array}{c}\mathrm{P}- \\
\text { value }\end{array}$ \\
\hline 5-Yr local recurrence free-survival $(\%)$ & 92.1 & 96.1 & 0.197 \\
5-Yr metastasis free-survival (\%) & 75.4 & 80.6 & 0.663 \\
5-Yr overall survival (\%) & 87.7 & 92.6 & 0.167 \\
5-Yr cancer specific-survival (\%) & 88.7 & 94.2 & 0.08 \\
\hline
\end{tabular}


A

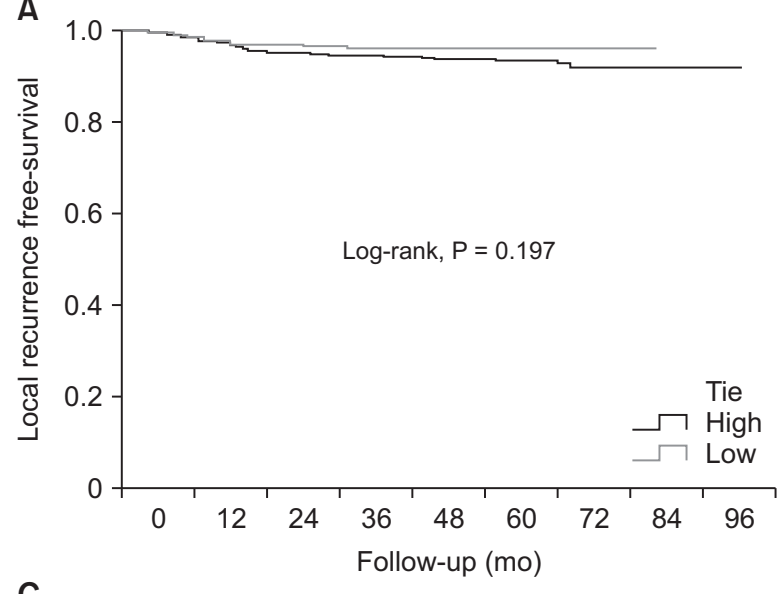

C

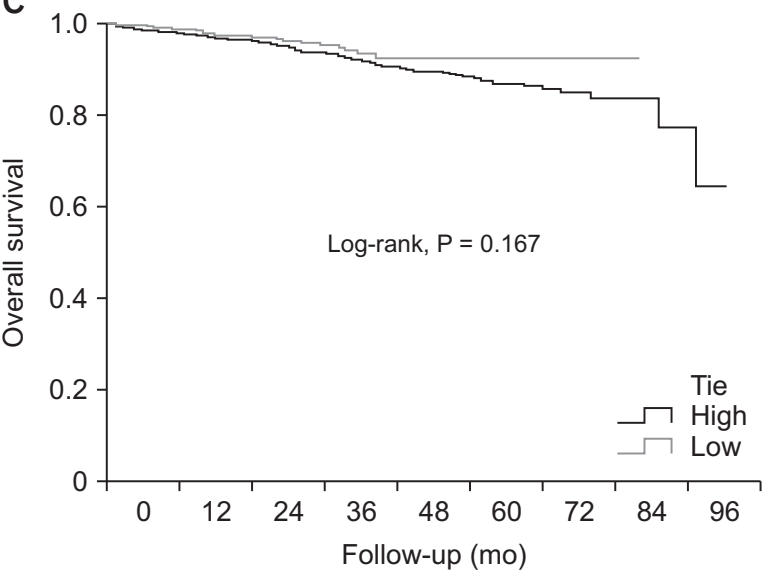

B

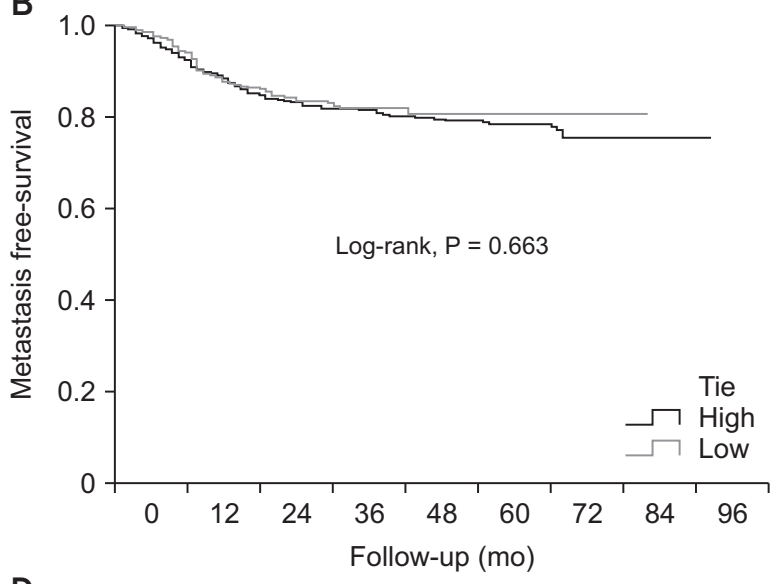

D

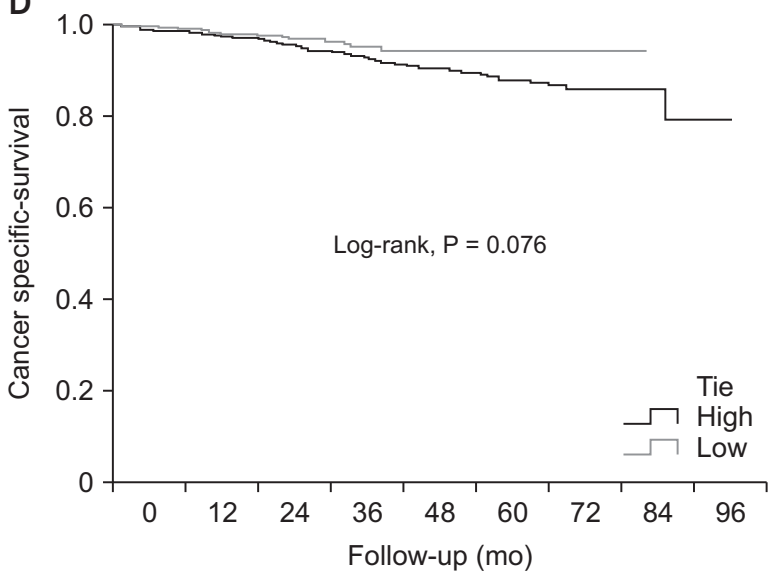

Fig. 2. Oncologic outcomes. (A) Local recurrence-free survival curves, (B) metastasis-free survival curves, (C) overall survival curves, and (D) cancer-specific survival curves of patients with rectal cancer treated with high-tie ligation or low-tie ligation with lymph node dissection.

oncologic outcomes [3]. Few studies have compared high IMA ligation and low IMA ligation in terms of both complication (especially AL) rates and oncologic outcomes [6,7]. Inadequate blood perfusion in the proximal limb of the colon and tension in the anastomosis are major contributing factors to AL and are generally associated with each other [15]. Dworkin and Allen-Mersh [16] investigated the disadvantages of high IMA ligation by using Doppler flowmetry, daily for 5 days after surgery, to assess the effects of clamping the IMA above the anastomosis in 26 patients. They found that clamping reduced the blood flow to the sigmoid colon by $50 \%$ during the first 5 postoperative days. In another study, Seike et al. [17] reported similar results. Moreover, in a retrospective study, Tsujinaka et al. [18] warned of potential necrosis of the proximal bowel after high IMA ligation. In their study, such necrosis developed in $2 \%$ of the patients who underwent high IMA ligation and in none of the patients who underwent low IMA ligation. However, high IMA ligation is often preferred over low IMA ligation to achieve a tension-free anastomosis, because high IMA ligation provides a longer colon length than does low IMA ligation, especially in cases of ultra-low LAR with straight or J-pouch coloanal anastomosis [19]. Several researchers have investigated how the level of IMA ligation affects the risk of AL development [20,21]. Tocchi et al. [20] studied the impact of IMA ligation on AL in 163 patients with diverticular disease of the sigmoid colon and found that IMA ligation resulted in a significantly increased rate of AL. In contrast, Rutegard et al. [21], in a large populationbased study, found that high IMA ligation did not result in an increased incidence of symptomatic AL. In our study, the rates of AL and other complications were not significantly different between patients who underwent high IMA ligation and those who underwent low IMA ligation, similar to the results of a previous RCT (HIGHLOW trial) [9]. However, as AL was the secondary outcome of this trial, there is a limitation in that the study was underpowered. The authors suggested that a future RCT tailored to this specific primary outcome should be conducted to provide reliable results.

Several studies have reported the oncologic outcomes of LN dissection around the origin of the IMA [22,23]. Kanemitsu et al. [22] reported that high IMA ligation prolonged survival in patients with colorectal cancer. Only $1.7 \%$ of the patients had metastases in LNs near the IMA; however, among those 
patients, dissection of the LNs up to the origin of the IMA resulted in a 5-year survival rate of $40 \%$. In contrast, Pezim and Nicholls [24] and Corder et al. [25] found no difference in survival between patients who underwent high IMA ligation and those who underwent low IMA ligation in rectal cancer surgery. In addition, there was no significant difference in terms of the development of metastatic disease between the groups in the HIGHLOW Trial, in which no positive apical LN was found in either group [9]. Our results also showed comparable oncologic outcomes between the high- and lowtie groups in terms of the rates of local and distant recurrencefree survival, 5-year overall survival, and 5-year cancer-specific survival. However, we found positive apical LNs in both groups, although there was no significant difference $(2.4 \%$ vs. $1.6 \%, \mathrm{P}=$ 0.16).

In several studies, fewer LNs were extracted when the LN dissection was limited to preserve of the origin of the IMA $[26,27]$, suggesting that high IMA ligation may allow more LNs to be harvested and thus increase the accuracy of tumor staging. We found that the numbers of harvested LNs and metastatic apical LNs were similar between patients who underwent high IMA ligation and those who underwent low IMA ligation, although patients who underwent high IMA ligation had more metastatic LNs on average and more advanced pathologic nodal stage. This may suggest that surgeons prefer high-tie ligation when an advanced nodal stage of apical LN metastasis is suspected on preoperative evaluation. Moreover, most metastatic LNs are intermediate or paracolic LNs, which may imply that low ties that preserve the autonomic nerve are oncologically safe and preferred with respect to genitourinary function. Mari et al. [9] concluded in their RCT that low ligation of the IMA in LAR results in better genitourinary function preservation without affecting the initial oncologic outcomes. The low-tie group showed better continence, less obstructive urinary symptoms, and improved postoperative quality. Sexual function was also better in the low-tie group than in the hightie group.

In laparoscopic resection of colorectal cancer, some surgeons preserve the IMA and LCA and dissect the LNs around the origin of the IMA, which is technically demanding and time consuming [28]. Accordingly, we observed that the operative time was significantly longer in low IMA ligation with LN dissection than in high IMA ligation. Furthermore, the branching of the IMA (LCA and sigmoid arteries) varies among individual patients [29], and an understanding of those variations is essential, especially for the low ligation technique. Importantly, whichever procedure is adopted (high or low IMA ligation), a meticulous technique is mandatory to obtain good short- and long-term outcomes.

In summary, we observed similar AL rates and oncologic outcomes between patients who underwent high IMA ligation and those who underwent low IMA ligation with dissection of the LNs around the origin of the IMA. However, this study is limited by its nonrandomized retrospective design, which may have caused a selection bias. The involvement of multiple surgeons might have also affected the outcomes; however, all surgeons were experienced colorectal surgeons and seemed to have used standardized operative techniques for low and high IMA ligation, as well as for splenic flexure mobilization in open or minimally invasive surgery. Another limitation is that we did not assess the patients' postoperative defecatory and genitourinary function. Compared with procedures involving low IMA ligation, procedures that involve high IMA ligation may be associated with poorer bowel function. Furthermore, in procedures involving high IMA ligation, the nerves arising from the root of the IMA are sacrificed and the supply of blood to the neorectum is reduced, which may cause impairment of defecatory function. In discordance with that hypothesis, Matsuda et al, reported that the level of IMA ligation did not have an effect on defecatory function [30].

In conclusion, low IMA ligation with dissection of the LNs around the origin of the IMA showed no differences in terms of AL rate compared with high IMA ligation, without affecting the oncologic outcomes. High IMA ligation did not seem to increase the number of total harvested LNs, whereas the ratio of metastatic apical LNs was similar between the 2 groups.

\section{CONFLICTS OF INTEREST}

No potential conflict of interest relevant to this article was reported.

\section{REFERENCES}

1. Beahrs OH. Staging of cancer of the colon and rectum. Cancer 1992;70(5 Suppl):13936.

2. Sugarbaker PH, Corlew S. Influence of sur- gical techniques on survival in patients with colorectal cancer. Dis Colon Rectum 1982;25:545-57.

3. Cirocchi R, Trastulli S, Farinella E, Desi- derio J, Vettoretto N, Parisi A, et al. High tie versus low tie of the inferior mesenteric artery in colorectal cancer: a RCT is needed. Surg Oncol 2012;21:e111-23. 
4. Hida J, Okuno K. High ligation of the inferior mesenteric artery in rectal cancer surgery. Surg Today 2013:43:8-19.

5. Lange MM, Buunen M, van de Velde CJ, Lange JF. Level of arterial ligation in rectal cancer surgery: low tie preferred over high tie. A review. Dis Colon Rectum 2008:51:1139-45.

6. Allison AS, Bloor C, Faux W, Arumugam P, Widdison A, Lloyd-Davies E, et al. The angiographic anatomy of the small arteries and their collaterals in colorectal resections: some insights into anastomotic perfusion. Ann Surg 2010;251:1092-7.

7. Komen N, Slieker J, de Kort P, de Wilt JH, van der Harst E, Coene PP, et al. High tie versus low tie in rectal surgery: comparison of anastomotic perfusion. Int J Colorectal Dis 2011;26:1075-8.

8. Surtees P, Ritchie JK, Phillips RK. High versus low ligation of the inferior mesenteric artery in rectal cancer. Br J Surg 1990; 77:618-21.

9. Mari GM, Crippa J, Cocozza E, Berselli M, Livraghi L, Carzaniga P, et al. Low ligation of inferior mesenteric artery in laparoscopic anterior resection for rectal cancer reduces genitourinary dysfunction: results from a randomized controlled trial (HIGHLOW Trial). Ann Surg 2018 Jul 10 [Epub]. https://doi.org/10.1097/ SLA.0000000000002947.

10. Chin CC, Yeh CY, Tang R, Changchien CR, Huang WS, Wang JY. The oncologic benefit of high ligation of the inferior mesenteric artery in the surgical treatment of rectal or sigmoid colon cancer. Int J Colorectal Dis 2008;23:783-8.

11. Edge SB, Compton CC. The American Joint Committee on Cancer: the 7th edition of the AJCC cancer staging manual and the future of TNM. Ann Surg Oncol 2010;17: 1471-4.

12. Nagtegaal ID, van de Velde CJ, van der Worp E, Kapiteijn E, Quirke P, van Krieken JH, et al. Macroscopic evaluation of rectal cancer resection specimen: clinical significance of the pathologist in quality control. J Clin Oncol 2002;20:1729-34.

13. Bae SU, Min BS, Kim NK. Robotic low ligation of the inferior mesenteric artery for rectal cancer using the firefly technique. Yonsei Med J 2015:56:1028-35.

14. Kim NK, Kim YW, Cho MS. Total mesorectal excision for rectal cancer with emphasis on pelvic autonomic nerve preservation: expert technical tips for robotic surgery. Surg Oncol 2015;24:172-80.

15. Bruch HP, Schwandner O, Schiedeck TH, Roblick UJ. Actual standards and controversies on operative technique and lymphnode dissection in colorectal cancer. Langenbecks Arch Surg 1999;384:167-75.

16. Dworkin MJ, Allen-Mersh TG. Effect of inferior mesenteric artery ligation on blood flow in the marginal artery-dependent sigmoid colon. J Am Coll Surg 1996;183: 357-60.

17. Seike K, Koda K, Saito N, Oda K, Kosugi C, Shimizu K, et al. Laser Doppler assessment of the influence of division at the root of the inferior mesenteric artery on anastomotic blood flow in rectosigmoid cancer surgery. Int J Colorectal Dis 2007; 22:689-97.

18. Tsujinaka S, Kawamura YJ, Tan KY, Mizokami K, Sasaki J, Maeda T, et al. Proximal bowel necrosis after high ligation of the inferior mesenteric artery in colorectal surgery. Scand J Surg 2012;101:21-5.

19. Bonnet S, Berger A, Hentati N, Abid B, Chevallier JM, Wind $\mathrm{P}$, et al. High tie versus low tie vascular ligation of the inferior mesenteric artery in colorectal cancer surgery: impact on the gain in colon length and implications on the feasibility of anastomoses. Dis Colon Rectum 2012; 55:515-21.

20. Tocchi A, Mazzoni G, Fornasari V, Miccini M, Daddi G, Tagliacozzo S. Preservation of the inferior mesenteric artery in colorectal resection for complicated diverticular disease. Am J Surg 2001;182:162-7.

21. Rutegard M, Hemmingsson O, Matthiessen P, Rutegard J. High tie in anterior resection for rectal cancer confers no increased risk of anastomotic leakage. $\mathrm{Br} \mathrm{J}$ Surg 2012;99:127-32.

22. Kanemitsu Y, Hirai T, Komori K, Kato T. Survival benefit of high ligation of the in- ferior mesenteric artery in sigmoid colon or rectal cancer surgery. Br J Surg 2006;93: 609-15.

23. Titu LV, Tweedle E, Rooney PS. High tie of the inferior mesenteric artery in curative surgery for left colonic and rectal cancers: a systematic review. Dig Surg 2008;25:14857.

24. Pezim ME, Nicholls RJ. Survival after high or low ligation of the inferior mesenteric artery during curative surgery for rectal cancer. Ann Surg 1984;200:729-33.

25. Corder AP, Karanjia ND, Williams JD, Heald RJ. Flush aortic tie versus selective preservation of the ascending left colic artery in low anterior resection for rectal carcinoma. Br J Surg 1992;79:680-2.

26. Kawamura YJ, Umetani N, Sunami E, Watanabe T, Masaki T, Muto T. Effect of high ligation on the long-term result of patients with operable colon cancer, particularly those with limited nodal involvement. Eur J Surg 2000;166:803-7.

27. Uehara K, Yamamoto S, Fujita S, Akasu T, Moriya Y. Impact of upward lymph node dissection on survival rates in advanced lower rectal carcinoma. Dig Surg 2007;24: 375-81.

28. Kobayashi M, Okamoto K, Namikawa T, Okabayashi T, Araki K. Laparoscopic lymph node dissection around the inferior mesenteric artery for cancer in the lower sigmoid colon and rectum: is D3 lymph node dissection with preservation of the left colic artery feasible? Surg Endosc 2006;20:563-9.

29. Adachi Y, Kakisako K, Sato K, Shiraishi N, Miyahara M, Kitano S. Factors influencing bowel function after low anterior resection and sigmoid colectomy. Hepatogastroenterology 2000;47:155-8.

30. Matsuda K, Hotta T, Takifuji K, Yokoyama S, Oku Y, Watanabe T, et al. Randomized clinical trial of defaecatory function after anterior resection for rectal cancer with high versus low ligation of the inferior mesenteric artery. Br J Surg 2015;102:5018. 